\section{AB0151 EXPRESSION OF ANXIETY AND DEPRESSION IN PATIENTS WITH ANKYLOSING SPONDILITE DEPENDING ON THE STATE OF THE AUTONOMIC NERVOUS SYSTEM AND INTENSITY OF THE PAIN SYNDROME}

Irina Blaginina ${ }^{1}$, Olga Rebrova ${ }^{1}$, Anna Blagodarenko ${ }^{1}$, Pavel Bakhtoyarov ${ }^{1}$, Olga Yavorskaya ${ }^{2}{ }^{1}$ Lugansk State Medical University, Lugansk, Ukraine;

${ }^{2}$ Lugansk republican clinical hospital, rheumatology, Lugansk, Ukraine

Background: Chronic pain syndrome, immanent in the majority of immuneinflammatory processes, is one of the pathophysiological mechanisms of the development of emotional and affective disorders. It has been proven that high levels of "pro-inflammatory" mediators are predictors of depression, and increased anxiety leads to an imbalance in the autonomic nervous system (ANS). It is also known that the pain syndrome worsens the autonomic supply of the organism. However, this problem remains insufficiently studied today.

Objectives: To assess the severity of anxiety and depression in patients with ankylosing spondilite, depending on the state of the ANS and pain syndrome. Methods: The study included 75 patients with ankylosing spondylitis (AS), who studied the frequency of anxiety-depressive disorders and their severity on the Spielberger anxiety and Hamilton depression scales. The autonomic status was investigated according to the methods "Wayne-patient" - WP, filled by the patient (more than 15 points - possible autonomic dysfunction (AD)) and "Wayne-doctor" - WD, filled by a doctor (more than 25 points - confirms the presence of signs of $A D)$; we evaluated the indicators of heart rate variability (HRV) - mode (Mo), mode amplitude (AMo), autonomic equilibrium index (AEI), regulatory systems tension index (TI), the standard deviation of the normal RR intervals (SDNN), the square root of the mean squares of the difference between successive RR intervals (RMSSD) and the balance ratio of the activity of the sympathetic and parasympathetic divisions of the ANS (LF/HF). Pain syndrome and the patient's own health index (POH) were assessed by a visual analogue scale (VAS) by patients, and by a physician - by counting the number of painful joints (NPJ), index BASDAI and ASDAS indices.

Results: In $25.3 \%$ of patients with AS, a high situational anxiety was observed and in $36 \%$ of patients high personal anxiety (PA) was observed. Depressive disorders were found in 57 patients with AS (76\%). The severity of pain in the joints and the spine influenced the increase in PA $(F=2.75, p=0.047 ; F=3.54, p=$ 0.037 , respectively) and the level of depressive disorders $(F=3.28, p=0.03 ; F=$ 3.44, $p=0.047$, respectively), and the level of depression is also $\mathrm{POH}(\mathrm{F}=3.49, \mathrm{p}$ $=0.048$ ). $68 \%$ patients had an AD (according to WP and WD methods). They constituted the 1 st group of observation, and the remaining 24 patients were included in the 2nd group. In group 1, signs of anxiety disorders were identified in $84.3 \%$ of cases, and in patients without AD in $45.8 \%$ of cases. Depressive disorders were observed in $94 \%$ patients with autonomic disorders (11 cases of psychotic, 37 non-psychotic depression) and in $37.5 \%$ in patients without signs of AD - signs of non-psychotic depression.

In both groups, there was a decrease in SDNN and RMSSD in comparison with normative ones, which indicates an increase in sympathetic regulation. In the $1 \mathrm{st}$ group, there was a more significant increase in LF/HF $(4.15 \pm 0.64)$ and a decrease in SDNN $(22.4 \pm 5.6 \mathrm{~ms})$ than in the $2 \mathrm{nd}(3.0 \pm 0.86 ; 29.1 \pm 8.4 \mathrm{~ms})$, which indicates the connection between the strengthening of the sympathetic regulation link and the presence of signs of psycho-emotional disorders. The growth of AMo in the 1 st group $(47.9 \pm 8.4 \%)$ reflects the degree of mobilizing influence of the sympathetic division and indicates an increase in the activation of the central contour and the growth of sympathetic regulation in patients with $A S$ with $A D$.

Conclusion: Patients with AS have a high incidence of clinically significant anxiety and depression, which were affected by the intensity of the pain syndrome. The presence of autonomic imbalance and dysregulation of the sympathetic link of the ANS also contribute to the strengthening of psycho-emotional disorders in this category of patients.

Disclosure of Interests: None declared

DOI: 10.1136/annrheumdis-2019-eular.1693

\section{AB0152 IL-23 INDUCES OSTEOCLASTS TO EXPRESS PRO- OSTEOGENIC FACTORS RATHER THAN ANTI- OSTEOGENIC FACTORS}

Dan-Dan Pang, LI Cai, Sheng-Ming Dai. Shanghai Jiao Tong University Affiliated Sixth People's Hospital, Shanghai, China

Background: The formation of new bone or syndesmophytes is the dominant factor of the damage caused by AS, but the mechanisms responsible for syndesmophyte formation in AS have not been elucidated.

Objectives: To explore whether IL-23 indirectly facilitates new bone formation through osteoclasts in ankylosing spondylitis, here we analyzed whether IL-23 enhances the expression levels of osteogenic factors by osteoclasts.

Methods: Mouse bone marrow mononuclear cells were cultured in the presence of M-CSF $(50 \mathrm{ng} / \mathrm{ml})$ and receptor activator of NF- $\lambda B$ ligand (RANKL, $30 \mathrm{ng} / \mathrm{ml})$ to induce osteoclast formation in vitro. The mRNA and protein expression levels of the osteogenesis-modulating factors, such as ephrin $\mathrm{B} 2$, sphingosine kinase 1 (SPHKI), bone morphogenetic protein 2 (BMP2), BMP6, Wnt10b, semaphorin 4D and $\mathrm{Htr} A 1$, were analyzed by quantitative real-time RT-PCR and Western blotting. Results: Mature osteoclasts were successfully induced from primary bone marrow mononuclear cells in the presence of both M-CSF and RANKL. Mature osteoclasts were identified by elevated expression levels of tartrate-resistant acid phosphatase (TRAP) and NFATc1, as well as positive TRAP staining with more than 3 nuclei. IL-23 increased the mRNA expression levels of pro-osteogenic ephrin B2, SPHKI and BMP2 in osteoclasts to $2.1,2.46$ and 1.46 folds, respectively. The elevated protein levels of ephrin B2 and SPHK1 in IL-23-stimulated osteoclasts were also confirmed by Western blotting. IL-23 failed to enhance the expression levels of pro-osteogenic BMP6 and Wnt10b and showed no effects on the expression levels of anti-osteogenic semaphorin 4D and HtrA1 in osteoclasts. Conclusion: IL-23 induces osteoclasts to express pro-osteogenic factors rather than anti-osteogenic factors, suggesting IL-23 may indirectly promote the differentiation of osteoblasts through osteoclasts in ankylosing spondylitis.

Disclosure of Interests: None declared

DOI: 10.1136/annrheumdis-2019-eular.764

\section{AB0153 CLINICAL SIGNIFICANCE OF MONOCYTE TO HEMOGLOBIN RATIO AND PLATELET TO ALBUMIN RATIO IN PATIENTS WITH AXIAL SPONDYLOARTHRITIS}

Yukai Huang, Qidang Huang, Meng Liu, Zheng Zhong, Fan Feng, Jiawei Jiang, Yuqi Liu, LI Tianwang. Guangdong Second Provincial General Hospital, Department of Rheumatology and Immunology, Guangzhou, China

Background: Axial spondyloarthritis (axSpA) is a progressive, chronic, inflammatory skeletal disorder. Platelet to hemoglobin ratio (PHR) and platelet to albumin ratio (PAR) have been recommended as prognostic factor of cancer However, none of study has focused on the clinical significance of PHR, PAR, neutrophil to hemoglobin ratio (NHR), neutrophil to albumin ratio (NAR), monocyte to hemoglobin ratio(MHR), monocyte to albumin ratio(MAR) for axSpA.

Objectives: The study aims to determine the clinical significance of NHR, NAR, MHR, MAR, PHR, PAR for axSpA.

Methods: A total of 198 axSpA patients and 48 healthy subjects were enrolled in the study retrospectively. Neutrophil, monocyte, platelet, hemoglobin, albumin, NHR, NAR, MHR, MAR, PHR, PAR, C-reactive protein (CRP), erythrocyte sedimentation rate (ESR) and Bath Ankylosing Spondylitis Disease Activity Index (BASDAI) were collected. AxSpA patients were divided into remission group $(B A S D A l<4)$ and active group(BASDAl $\geq 4)$. Relationships between the parameters and BASDAI were assessed by the Sperman's correlations analysis. Receiver operation characteristic (ROC) curves were used to discriminate axSpA patients from healthy subjects and active group from remission group.

Results: Neutrophil, monocyte, platelet, NHR, NAR, MHR, MAR, PHR, PAR, CRP, ESR in axSpA group were higher than those of control group, while albumin and hemoglobin were lower $(P<0.05)$. ROC curve results showed that the AUC value of MHR for axSpA were 0.808 (C195\%: 0.738-0.878), yielding a highest AUC value than other parameters. The optimal cutoff value of MHR for axSpA was 0.002447 , with the Youden index of 0.498 , sensitivity of $87.4 \%$ and specificity of $62.5 \%$. The closely positive correlations were found between PAR and BASDAI $(r=0.379, P<0.001)$. Besides, platelet, NAR, MAR, PHR, PAR, CRP, ESR in active group were higher than those of remission group, while albumin were lower $(P<0.05)$. ROC curve results showed that the AUC value of PAR for active group were 0.693 (Cl95\%: 0.610-0.775), yielding a highest AUC value than other parameters. The optimal cutoff value of PAR for active group was 5.967 , with the Youden index of 0.354 , sensitivity of $83.3 \%$ and specificity of $52.1 \%$.
A

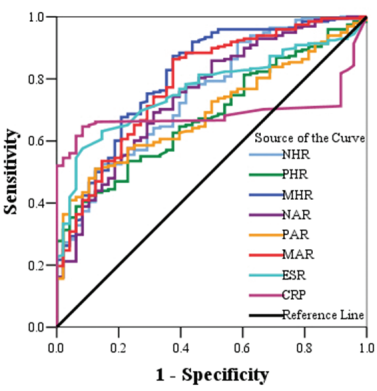

B

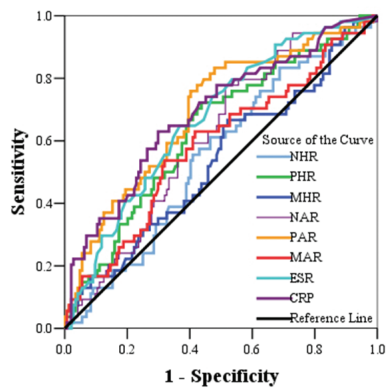

Figure.1 ROC curves were used to discriminate axSpA patients from healthy subjects(A) and active group from remission group(B). 